\title{
Pneumonia de hipersensibilidade subaguda: ocorrência em familiares
}

\author{
Sub acute hypersensitivity pneumonitis: incidence among family members
}

Danila de Souza Carraro ${ }^{1}$, Gilberto Petty da Silva², Clóvis Eduardo T. Gomes ${ }^{3}$

\section{RESUMO}

Objetivo: Descrever um caso de pneumonia de hipersensibilidade subaguda em criança com ocorrência concomitante da mesma doença em um familiar.

Descrição do caso: Paciente de 12 anos encaminhado para investigação de dispnéia progressiva, tosse seca e emagrecimento. Realizou vários tratamentos por hipótese de pneumonias bacterianas de repetição. Após diagnóstico radiológico e histopatológico de pneumonia de hipersensibilidade, apresentou melhora clínica com remoção da exposição. A mãe do paciente foi internada no mesmo período com quadro clínico-radiológico e diagnóstico semelhantes.

Comentários: A pneumonia de hipersensibilidade representa um grupo de doenças caracterizadas por processo inflamatório, que compromete o parênquima pulmonar e as vias aéreas em resposta a uma exposição a grande variedade de antígenos inalados. Pode se apresentar sob as formas aguda, subaguda ou crônica. Sua incidência varia de acordo com aspectos ambientais e geográficos. Somente $25 \%$ dos pacientes têm histórico familiar. A existência de predisposição genética para a doença ainda é desconhecida.

Palavras-chave: pneumonia de hipersensibilidade; criança; família.

\section{ABSTRACT}

Objective: To describe a case of subacute hipersensitivity pneumonitis in a boy with simultaneous occurrence of the same clinical syndrome in a member of his family.

Case description: A 12 years old child with worsening dyspnea, dry cough and weigth loss. He had no improvement despite several treatments for bacterial pneumonia. The diagnosis of hypersensitivity pneumonia was made based on radiologic and hystophatologic patterns, and the patient got better after removal of exposure. The mother of the patient had a similar clinical and radiologic disease at the same period.

Comments: The hypersensitivity pneumonitis represents a group of diseases characterized by inflammatory damage of lung parenchyma and airways in response to inhalation of a large variety of antigens. The clinical presentation varies with acute, subacute and chronic forms. Its incidence also varies considerably, depending on environmental and geographic conditions. Only 25\% of cases have a family history of the disease. The existence of an exact genetic risk factor is still unknown.

Key-words: pneumonitis hypersensitivity; child; family
Instituição: Hospital São Paulo da Universidade Federal de São Paulo (Unifesp), São Paulo, SP, Brasil

${ }^{1}$ Médica residente do setor de Pneumologia Pediátrica do Departamento de Pediatria da Unifesp, São Paulo, SP, Brasil

${ }^{2}$ Professor adjunto do setor de Pneumologia Pediátrica do Departamento de Pediatria da Unifesp, São Paulo, SP, Brasil.

${ }^{3}$ Professor adjunto e chefe do setor de Pneumologia Pediátrica do Departamento de Pediatria da Unifesp, São Paulo, SP, Brasil
Endereço para correspondência:

Danila de Souza Carraro

Rua Diogo de Farias, 307

CEP 04037-000 - São Paulo/SP

E-mail: danilamed@ig.com.br

Recebido em: 13/3/2008

Aprovado em: 26/7/2008 


\section{Introdução}

A pneumonia de hipersensibilidade é uma doença inflamatória do parênquima pulmonar de natureza imunológica causada pela exposição e inalação de poeiras orgânicas ou substâncias químicas ${ }^{(1)}$. Foi descrita em poucas crianças até o momento e somente $25 \%$ dos pacientes possuem caso familiar concomitante $e^{(1)}$.

A incidência varia de acordo com o país. Apenas 5 a $15 \%$ dos indivíduos expostos desenvolvem a síndrome clínica; há, ainda, a possibilidade de existir predisposição genética que determine quem irá adoecer ${ }^{(2)}$. Há três diferentes apresentações clínicas para a pneumonia de hipersensibilidade: aguda, subaguda e crônica ${ }^{(3)}$.

Neste relato apresentamos um caso de pneumonia de hipersensibilidade subaguda e uma breve discussão sobre o assunto.

\section{Descrição do caso}

Paciente masculino de 12 anos, procedente de Cubatão, previamente hígido. Há dois meses mostrava dispnéia progressiva aos esforços, tosse seca, anorexia e perda de peso. No inicio do quadro apresentou febre diária durante uma

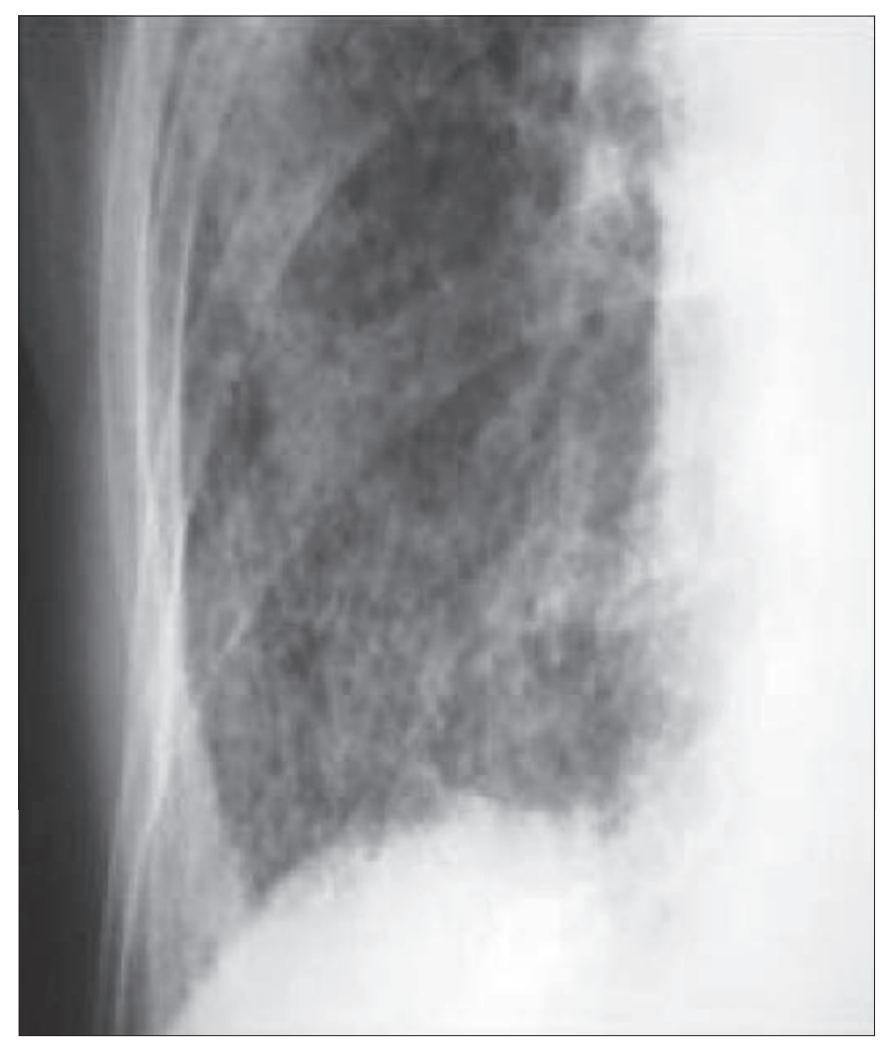

Figura 1 - Infiltrado micronodular difuso bilateral. semana. Fez uso de três esquemas antimicrobianos antes de procurar o serviço. Suas condições de moradia eram precárias, com presença de mofo nas paredes de todos os cômodos.

O paciente foi internado na enfermaria de Pneumologia Pediátrica do Hospital São Paulo para investigação diagnóstica. $\mathrm{Na}$ ausculta respiratória, apresentava estertores crepitantes em bases, oximetria de pulso com $\mathrm{SpO}_{2} 98 \%$ no repouso e queda com pico máximo de $\mathrm{SpO}_{2} 90 \%$ durante o exercício. Hemograma e nível IgE sérica apresentaram-se dentro da normalidade e não foram solicitadas precipitinas séricas ou testes de anticorpos específicos do tipo IgE (RAST). O paciente não evidenciava epidemiologia para tuberculose. A avaliação da função pulmonar por espirometria demonstrava distúrbio ventilatório sugestivo de processo restritivo: capacidade vital funcional (CVF) 1,7L (61\%), volume expiratório forçado no primeiro segundo (VEF1) 1,4L (57\%) e relação VEF1/CVF 90\%.

A radiografia de tórax (Figura 1) evidenciava infiltrado intersticial micronodular difuso bilateral; a tomografia de alta resolução do tórax (Figura 2) apresentava infiltrado em "vidro fosco" bilateral, com micronódulos centrilobulares.

A análise citológica diferencial do lavado broncoalveolar mostrava 90\% de macrófagos alveolares, $5 \%$ de linfócitos e $5 \%$ de neutrófilos. A pesquisa e cultura para bactérias, fungos e micobactérias tiveram resultado negativo. A biópsia pulmonar a céu aberto (Figura 3 e 4) levou ao achado de alveolite e infiltrado intersticial de distribuição predominante peribronquiolar com predomínio de linfócitos, formação de granulomas não-caseosos e bronquíolos preenchidos por



Figura 2 - Nódulos centrilobulares em vidro fosco. 


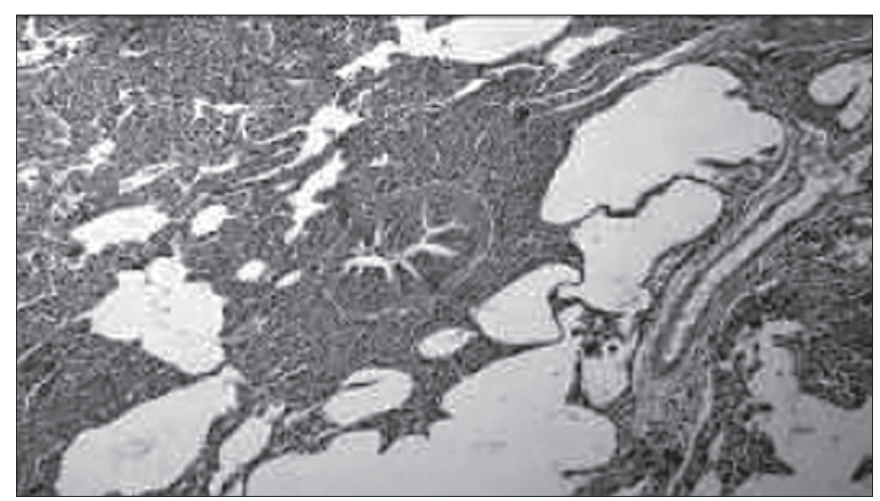

Figura 3 - Lesão bronquiolocêntrica representada por inflamação linfoplasmocitária, HE (100X).

células inflamatórias. A pesquisa de bacilo álcool-ácido resistente (BAAR) e fungos apresentou resultado negativo. O diagnóstico histológico foi feito com base nos achados clássicos da pneumonia de hipersensibilidade descritos por Katzenstein $^{(18)}$.

A associação de dados clínicos, epidemiológicos, radiológicos e, sobretudo, de biópsia pulmonar, indicou o diagnóstico de hipersensibilidade pulmonar em fase subaguda. Após dois meses de afastamento do domicílio, o paciente evoluiu com melhora clínica progressiva, com resolução total dos sintomas respiratórios e sem necessidade de corticoterapia. O teste de caminhada não mais evidenciava queda da saturação de oxigênio durante o esforço $\left(\mathrm{SpO}_{2} 95 \%\right)$. As imagens radiológicas não foram repetidas até o momento.

A mãe do paciente foi internada no mesmo período com quadro clínico-radiológico semelhante. Diagnosticou-se pneumonia de hipersensibilidade pelo mesmo fator de exposição no domicílio, sem necessidade de biópsia pulmonar. A paciente apresentou melhora clínica após remoção da exposição e corticoterapia por 30 dias.

\section{Comentários}

A pneumonia de hipersensibilidade é uma síndrome clínica complexa, desencadeada pela resposta imunológica à inalação de antígenos. Pode se apresentar sob as formas aguda, subaguda e crônica, dependendo da quantidade, duração da exposição, natureza do antígeno e da reposta imunológica do paciente ${ }^{(4)}$. Desconhece-se a existência de predisposição genética ou familiar para a ocorrência da doença, não sendo identificada expressão gênica ou tipagem HLA com freqüência aumentada na doença, apesar de nem todos os indivíduos expostos ao mesmo antígeno desenvolverem sintomas ${ }^{(5)}$.

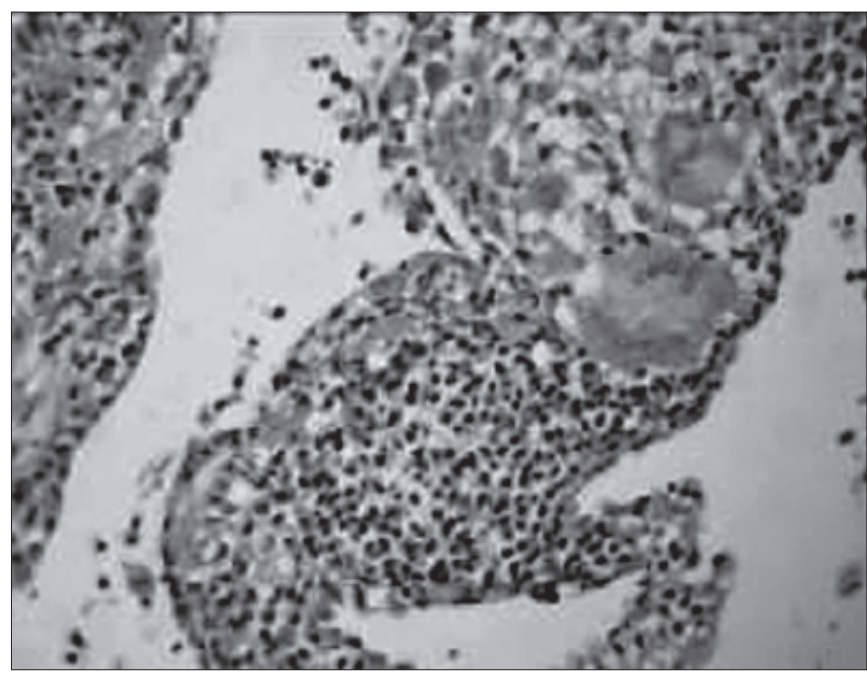

Figura 4 - Inflamação linfoplasmocitária permeada por células gigantes multinucleadas, HE (200X).

A forma aguda ocorre entre quatro e oito horas após a exposição; a forma subaguda e crônica durante semanas ou meses. A forma subaguda é caracterizada por início insidioso, dispnéia aos esforços, fadiga, tosse, emagrecimento e, na maioria dos casos, associa-se a inalantes domiciliares ${ }^{(6,7)}$. Há uma predominância da doença em pacientes do sexo masculino e a idade média do início dos sintomas na infância é dez anos ${ }^{(4)}$.

A pneumonia de hipersensibilidade ainda é pouco diagnosticada em nosso meio. É de extrema importância que seu diagnóstico seja feito precocemente e de acordo com a história de exposição, dados clínicos, achados radiológicos, função pulmonar e, se possível, por meio da resolução clínica após a remoção da potencial exposição do paciente. O lavado broncoalveolar e a biópsia pulmonar são métodos úteis para afastar outras patologias, além de reunirem dados para a conclusão diagnóstica ${ }^{(8-10)}$.

A pesquisa de precipitinas séricas, anticorpos do tipo IgG específicos contra determinados antígenos inalantes comuns e os testes de sensibilidade cutânea a tais antígenos têm valor questionável no diagnóstico da pneumonia de hipersensibilidade pois apresentam baixos valores de sensibilidade e especificidade ${ }^{(11)}$.

A tomografia torácica de alta resolução possui maior sensibilidade para detecção de achados sugestivos de pneumonia de hipersensibilidade do que a radiografia de tórax. Os principais padrões observados ao exame tomográfico incluem nódulos centrilobulares difusos ou focais, vidro fosco, padrão em mosaico, fibrose, consolidações peribrônquicas e cistos pulmonares $^{(12)}$. 
O lavado broncoalveolar com presença de grande percentual de linfócitos e de uma relação entre linfócitos T CD4 e CD8 inferior a um tende a colaborar com o diagnóstico de pneumonia de hipersensibilidade ${ }^{(13)}$. Nos casos de manifestação aguda, pode ocorrer aumento da população de eosinófilos.

Os achados histológicos nem sempre são conclusivos, porém a presença de pneumonia intersticial com acentuação peribronquiolar, bronquiolite obliterante e granulomas mal formados não necrosantes são a tríade histológica clássica dessa doença ${ }^{(14)}$.

O tratamento consiste no afastamento do agente desencadeante e, em alguns casos, de corticoterapia com prednisona $0,5-1 \mathrm{mg} / \mathrm{kg}$ (não ultrapassar $60 \mathrm{mg} / \mathrm{dia}$ ). A dose é mantida durante uma ou duas semanas e, em seguida, reduzida gradualmente até a interrupção. Estudos mostram redução mais

\section{Referências bibliográficas}

1. Fink JN, Ortega HG, Reynolds HY, Cormier YF, Fan LL, FranksTJ et al. Needs and Opportunities for Research in Hypersensitivity Pneumonitis. Am J Respir Crit Care Med 2005; 171:792-8.

2. Zamboni M, Pereira CA. Pneumologia: diagnóstico e tratamento. São Paulo: Atheneu; 2007.

3. Girard M, Israel-Assayag E, Cormier Y. Pathogenesis of hypersensitivity pneumonitis. Curr Opin Allergy Clin Immunol 2004;4:93-8.

4. Fan LL. Hypersensitivity Pneumonitis in Children. Curr Opin Pediatr 2002;4:323-6.

5. Wilson BD, Sternick JL, Yoshisawa Y, Katzeinstein AL, Moore VL. Genetic control of experimental murine hypersensitivity pneumonitis. Chest 1983;83(suppl 5):8S-9.

6. Schuyler M, Gott K, Cherne A. Mediators of hypersensitivity pneumonitis. J Lab Clin Med 2000;136:29-38.

7. Bertorelli G, Bocchino V, Olivieri D. Hypersensitivity pneumonitis. Eur Respir Mon 2000;14:120-36.

8. Matar LD, McAdams HP, Sporn TA. Hypersensitivity pneumonitis. Am J Roentgenol 2000;174:1061-6.

9. Schuyler M, Cormier Y. The diagnosis of hypersensitivity pneumonitis. Chest 1997;111:534-6.

10. Lacasse Y, Selman M, Costabel U, Dalphin JC, Ando M, Morell F et al. Clinical diagnosis of hypersensitivity pneumonitis. Am J Respir Crit Care Med 2003;168:952-8. rápida da sintomatologia com a corticoterapia, mas esta não exerce influência no curso da doença ${ }^{(15)}$.

O prognóstico da pneumonia de hipersensibilidade depende do antígeno envolvido, da resposta individual e da duração da exposição. Há poucos estudos que relatam acompanhamento a portadores de pneumonia de hipersensibilidade. O melhor prognóstico depende da intervenção médica precoce $e^{(16,17)}$.

\section{Agradecimento}

Agradecimento ao professor adjunto doutor do Departamento de Patologia da Universidade Federal de São Paulo (Unifesp), Rimarcs Gomes Ferreira, pelas imagens cedidas e laudo da biópsia pulmonar.

11. Cormier, Y, Lacasse, Y. Keys to the diagnosis of hypersensitivity pneumonitis: The role of serum precipitins, lung biopsy, and high-resolution computed tomography. Clin Pulm Med 1996;3:72.

12. Lynch DA, Rose CS, Way D, King TE Jr. Hypersensitivity pneumonitis: sensitivity of high resolution CT in a population-based study. Am J Roentgenol 1992;159:469-72.

13. Murayama J, Yoshizawa Y, Ohtsuka M, Hasegawa S. Lung fibrosis in hypersensitivity pneumonitis: association with CD4+ but not CD8+ cell dominant alveolitis and insidious onset. Chest 1993;104:38-43

14. Coleman A, Colby TV. Histologic diagnosis of extrinsic allergic alveolitis.Am J Surg Pathol 1988;12:514-8.

15. Kokkarinen JI, Tukiainen HO, Terho EO. Effect of corticosteroid treatment on the recovery of pulmonary function in farmer's lung. Am Rev Respir Dis 1992;145:3-5

16. Cuthbert OD, Gordon MF. Ten year follow-up of farmers with farmer's lung. $\mathrm{Br}$ J Ind Med 1983;40:173-6.

17. Vourlekis JS, Schwarz MI, Cherniack RM, Curran-Everett D, Cool CD, Tuder $\mathrm{RM}$ et al. The effect of pulmonary fibrosis on survival in patients with hypersensitivity pneumonitis. Am J Med 2004;116:662-8.

18. Katzenstein AL, Myers JL. Idiopathic pulmonary fibrosis: clinical relevance of pathologic classification. Am J Respir Crit Care Med 1998; 157(4 Pt 1): 1301-15. 